Journal of Mathematics and Statistics 8 (3): 403-412, 2012

ISSN 1549-3644

(C) 2012 Science Publications

\title{
Kernel Type Estimator and Statistical Properties for Intensity Function of Periodic Poisson Process with Power Function Trend
}

\author{
Ro'fah Nur Rachmawati \\ Department of Statistics, \\ School of Computer Science, Bina Nusantara University, Indonesia
}

\begin{abstract}
Problem statement: In this study, we construct the estimation for a periodic component of the intensity function of a periodic Poisson process in the presence of power function trend by using the general kernel function. Beside that we also construct the statistical properties of the estimator. Approach: It is considered the worst case where there is only available a single realization of the Poisson process having intensity which consist of a periodic component and a power function trend, observed in the interval $[0, \mathrm{n}]$. It is assumed that the period of the periodic component and the slope of the power function trend are known. Results: It has been formulated the estimator and asymptotic approximations to the bias and variance of the estimator. Conclusion: The estimator that we construct is asymptotically unbiased estimator for a periodic component of the intensity function of a periodic Poisson process in the presence of a power function trend.
\end{abstract}

Key words: Unbiased estimator, intensity function, several nonparametric methods, asymptotic normality, statistical properties

\section{INTRODUCTION}

On a periodic Poisson process, there are several nonparametric methods to estimate the function of the intensity at a given point, the methods are kernel type estimation and nearest neighbor estimation. These methods have been used to estimate the function of local intensity function with the period $\tau$ is known (Helmers and Mangku, 2000). In addition, the local intensity function estimation using the nearest neighbor method and the proofs of weak and strong consistency of the estimator obtained have been studied (Mangku, 1999). Another method is imitating the general form of maximum likelihood to estimate the rate of homogeneous Poisson process that can be applied to estimate the global intensity function in the periodic Poisson process (Helmers and Mangku, 2000). The intensity function of Poisson process has been used in modeling the rate of oil spill in the North Sea, Netherland (Helmers, 1995). Computationally, has been formulated an algorithm to estimate the intensity function of a Poisson process with exponential quadratic and periodic trends (Helmers and Zitikis, 1999).

Estimation of the intensity function can be distinguished based on the period, the period is known and the period is unknown. For the period is unknown, estimation of the intensity function is more complicated than process with the period is known. The consistency of kernel type estimator of the intensity function for periodic Poisson processes with unknown period have been studied (Helmers et al., 2003). For the period is known, the formulation of kernel type estimator and the proofs of weak and strong convergence of the estimator have been studied and the proof of asymptotic normality has been studied.

Modeling of a phenomenon with periodic Poisson process evolved to include a linear trend component (Helmers and Mangku, 2009) and using the double periodic intensity function (Helmers et al., 2007). One particular form of stochastic process with continuous time is a Poisson process with the intensity of a periodic function. This process can be used to model similar phenomena. If the arrival rate is increased by a power function of time, the most appropriate model to use is a periodic Poisson process with power function trend. In addition, the estimation for the intensity function of a periodic Poisson process that includes power function trend has been performed. The consistency of the estimator obtained using a uniform kernel function (Rahayu, 2008), statistical properties of the estimator obtained by using a uniform kernel function have been studied (Rachmawati, 2008) and the global intensity function estimation of periodic components also have been studied (Yuliawati, 2008).

In this study we will construct an estimator for a periodic component that includes power function trend, with the contribution of this study is in the estimation process we will use general kernel function, where the 
kernel function to be used is a function not only for uniform distribution but can be used for arbitrary distribution. By using a more general kernel function, the phenomena that can be presented by this model will more extensive and closer to the real phenomena because assumption that the event must have uniform distribution have been removed.

\section{MATERIALS AND METHODS}

Assumptions: Suppose $\mathrm{N}$ is a non homogeneous Poisson process on the interval $[0, \infty)$ with the intensity function $\lambda$ is unknown. This function is assumed to be locally integrable and consist of two components, a periodic (cyclic) component with period $\tau>0$ is known and a power function trend. In other words, for any point $s \in[0, \infty)$ the intensity function $\lambda$ can be written as follows Eq. 1:

$\lambda(s)=\lambda_{c}(s)+a s^{b}$

$\mathrm{a}>0$ and $0<\mathrm{b}<1$, with $\lambda_{\mathrm{c}}$ (s) is a periodic function with period $\tau$ and a is the slope of the trend and it is assumed that the value of $a, b$ and $\tau$ are known. This study does not assume a parametric form of $\lambda_{c}$ we suppose that only a single realization of the cyclic Poisson point process is observed within a bounded "window" (Mangku, 1999). Then, $\lambda_{\mathrm{c}}$ is periodic with the Eq. 2:

$$
\lambda_{\mathrm{c}}(\mathrm{s})=\lambda_{\mathrm{c}}(\mathrm{s}+\mathrm{k} \tau)
$$

for every $\in[0, \infty)$ and $k \in \mathbb{Z}$.

Suppose that for some $\omega \in \Omega$, there is only a single realization of $\mathrm{N}(\omega)$ of the Poisson process $\mathrm{N}$ defined on a probability space $(\Omega, \mathrm{F}, \mathrm{P})$ with the intensity function as in (1) was observed in the finite interval [0, $\mathrm{n}] \in[0, \infty)$. Because $\lambda_{\mathrm{c}}$ is periodic function with the period $\tau$, then the problem for estimating $\lambda_{\mathrm{c}}$ at the point $\mathrm{s}$, with $\mathrm{s} \in \mathbb{R}$ can be reduced to the problem estimating $\lambda_{\mathrm{c}}$ at the point $\mathrm{s}$, with $\mathrm{s} \in[0, \tau)$

A point $\mathrm{s}$, is called Lebesgue points of a function of $\lambda$ if Eq. 3 (Wheeden and Zygmund, 1977):

$$
\lim _{\mathrm{h} \rightarrow 0} \frac{1}{2 \mathrm{~h}} \int_{-\mathrm{h}}^{\mathrm{h}}|\lambda(\mathrm{x}+\mathrm{s})-\lambda(\mathrm{s})| \mathrm{dx}=0
$$

In this study, it is assumed that $\mathrm{s}$ is a Lebesgue point of $\lambda_{c}$. Suppose $h_{n}$, is a sequence of positive real numbers converging to zero Eq. 4 : $\mathrm{h}_{\mathrm{n}} \downarrow 0$

For $\mathrm{n} \rightarrow \infty$ and suppose $\mathrm{k}: \mathbb{R} \rightarrow \mathbb{R}$ called kernel function if it satisfies the following properties:

$(\mathrm{K} .1) \mathrm{k}=\mathrm{A}$ probability density function,

(K.2) $\mathrm{k}=$ Bounded,

(K.3) $\mathrm{k}=$ Has support in $[-1,1]$

The estimator: With the above assumptions, estimator for $\lambda_{c}$ at a given point $s, s \in[0, \tau)$ can be formulated as follows Eq. 5 and 6:

$$
\begin{aligned}
& \bar{\lambda}_{\mathrm{c}, \mathrm{n}, \mathrm{K}}(\mathrm{s})=\frac{1}{\mathrm{~L}_{\mathrm{n}, \mathrm{b}} \mathrm{h}_{\mathrm{n}}} \sum_{\mathrm{k}=1}^{\mathrm{n} \tau} \frac{1}{\mathrm{k}_{\mathrm{b}}} \int_{0}^{\mathrm{n}} \mathrm{K}\left(\frac{\mathrm{x}-(\mathrm{s}+\mathrm{k} \tau)}{\mathrm{h}_{\mathrm{n}}}\right) \mathrm{N}(\mathrm{dx}) \\
& -\frac{\mathrm{a}}{\mathrm{L}_{\mathrm{n}, \mathrm{b}}} \sum_{\mathrm{k}=1}^{\mathrm{n}_{\tau}} \frac{(\mathrm{s}+\mathrm{k} \tau)^{\mathrm{b}}}{\mathrm{k}^{\mathrm{b}}} \\
& \text { with } \mathrm{L}_{\mathrm{n}, \mathrm{b}}=\sum_{\mathrm{k}=1}^{\mathrm{n}_{\tau}} \frac{1}{\mathrm{k}^{\mathrm{b}}} \text { and } \mathrm{n}_{\tau}=\left[\frac{\mathrm{n}}{\tau}\right]
\end{aligned}
$$

The idea behind the estimation of kernel type estimator $\bar{\lambda}_{\mathrm{c}, \mathrm{n}, \mathrm{k}}$ of $\lambda_{\mathrm{c}}$ can be explained as follows:

From (1) and (2), for every point $s$ and $k \in \mathbb{Z}$ then:

$$
\lambda_{\mathrm{c}}(\mathrm{s})=\lambda_{\mathrm{c}}(\mathrm{s}+\mathrm{k} \tau)=\lambda(\mathrm{s}+\mathrm{k} \tau)-\mathrm{a}(\mathrm{s}+\mathrm{k} \tau)^{\mathrm{b}}
$$

With (6), the above equation can be written as Eq. 7:

$$
\begin{aligned}
& \lambda_{c}(s)=\frac{1}{L_{n, b}} \sum_{k=1}^{n_{\tau}} \frac{1}{k^{b}}\left(\lambda\left(s+k_{\tau}\right)-a(s+k \tau)^{b}\right) \\
& =\frac{1}{L_{n, b}} \sum_{k=1}^{n_{\tau}} \frac{1}{k^{b}}\left(\lambda(s+k \tau)-\frac{a}{L_{n, b}} \sum_{k=1}^{n_{\tau}} \frac{(s+k \tau)^{b}}{k^{b}}\right.
\end{aligned}
$$

Value of the function $\lambda(s+k \tau)$ at the point $s$, can be approximated by the average value of the number of events accured around $s$, in interval $\left[s+k \tau-h_{n}, s+k \tau+h_{n}\right]$ and by using (3), (7) can be written as Eq. 8:

$\bar{\lambda}_{\mathrm{c}, \mathrm{n}, \mathrm{K}}(\mathrm{s})$

$\approx \frac{1}{\mathrm{~L}_{\mathrm{n}, \mathrm{b}}} \sum_{\mathrm{k}=1}^{\mathrm{n}_{\tau}} \frac{1}{\mathrm{k}^{\mathrm{b}}} \frac{\mathrm{EN}\left(\left[\mathrm{s}+\mathrm{k} \tau-\mathrm{h}_{\mathrm{n}}, \mathrm{s}+\mathrm{k} \tau+\mathrm{h}_{\mathrm{n}}\right]\right)}{2 \mathrm{~h}_{\mathrm{n}}}$

$-\frac{1}{L_{n, b}} \sum_{k=1}^{n_{\tau}} \frac{(s+k \tau)^{b}}{k^{b}}$

By replacing EN $\left(\left[s+k \tau-h_{n}, s+k \tau+h_{n}\right]\right)$ with its stochastically equivalent $\mathrm{N}\left(\left[\mathrm{s}+\mathrm{k} \tau-\mathrm{h}_{\mathrm{n}}, \mathrm{s}+\mathrm{k} \tau+\mathrm{h}_{\mathrm{n}}\right]\right)$ so that (8) can be written as Eq. 9: 


$$
\begin{aligned}
& \bar{\lambda}_{c, n, \mathrm{~K}}(\mathrm{~s}) \\
& \approx \frac{1}{\mathrm{~L}_{\mathrm{n}, \mathrm{b}}} \sum_{\mathrm{k}=1}^{\mathrm{n}_{\varepsilon}} \frac{1}{\mathrm{k}^{\mathrm{b}}} \frac{1 \mathrm{~N}\left(\left[\mathrm{~s}+\mathrm{k} \tau-\mathrm{h}_{\mathrm{n}}, \mathrm{s}+\mathrm{k} \tau+\mathrm{h}_{\mathrm{n}}\right]\right)}{2 \mathrm{~h}_{\mathrm{n}}} \\
& -\frac{\mathrm{a}}{\mathrm{L}_{\mathrm{n}, \mathrm{b}}} \sum_{\mathrm{k}=1}^{\mathrm{n}_{\mathrm{n}}} \frac{(\mathrm{s}+\mathrm{k} \tau)^{\mathrm{b}}}{\mathrm{k}^{\mathrm{b}}} \\
& =\frac{1}{\mathrm{~L}_{\mathrm{n}, \mathrm{b}} \mathrm{h}_{\mathrm{n}}} \sum_{\mathrm{k}=1}^{\mathrm{n}_{\varepsilon}} \frac{1}{\mathrm{k}^{\mathrm{b}}} \int_{0}^{\mathrm{n}} \frac{1}{2} \mathrm{I}_{[-1,1,1}\left(\left[\mathrm{s}+\mathrm{k} \tau-\mathrm{h}_{\mathrm{n}}, \mathrm{s}+\mathrm{k}\right.\right. \\
& \left.\left.+\mathrm{h}_{\mathrm{n}}\right]\right) \mathrm{N}(\mathrm{dx})-\frac{\mathrm{a}}{\mathrm{L}_{\mathrm{n}, \mathrm{b}}} \sum_{\mathrm{k}=1}^{\mathrm{n}_{\mathrm{n}}} \frac{(\mathrm{s}+\mathrm{k} \tau)^{\mathrm{b}}}{\mathrm{k}^{\mathrm{b}}} \\
& =\frac{1}{\mathrm{~L}_{\mathrm{n}, \mathrm{b}} \mathrm{h}_{\mathrm{n}}} \sum_{\mathrm{k}=1}^{\mathrm{n} \tau} \frac{1}{\mathrm{k}^{\mathrm{b}}} \int_{0}^{\mathrm{n}} \mathrm{k}_{1}\left(\frac{\mathrm{x}-(\mathrm{s}+\mathrm{k} \tau)}{\mathrm{h}_{\mathrm{n}}}\right) \mathrm{N}(\mathrm{dx}) \\
& -\frac{\mathrm{a}}{\mathrm{L}_{\mathrm{n}, \mathrm{b}}} \sum_{\mathrm{k}=1}^{\mathrm{n} \tau} \frac{(\mathrm{s}+\mathrm{k} \tau)}{\mathrm{k}^{\mathrm{b}}}
\end{aligned}
$$

with $\mathrm{k}_{1}=\frac{1}{2}[-1,1]$. To generalized the estimator we used general kernel function $\mathrm{k}$.

\section{RESULTS}

\section{Statistical properties:}

Theorem 1: Asymptotic approximation of the expected value for the estimator: Suppose that the intensity function $\lambda$ as (1) and locally integrable. If the kernek $\mathrm{K}$ is symmetric and satisfies the conditions (K.1), (K.2), (K.3), $\mathrm{h}_{\mathrm{n}} \downarrow 0, \mathrm{n}^{1-2 \mathrm{~b}} \mathrm{~h}_{\mathrm{n}}{ }^{2} \rightarrow \infty$ for $\mathrm{n} \rightarrow \infty$ then Eq. 10:

$$
\begin{aligned}
& \mathrm{E} \bar{\lambda}_{\mathrm{c}, \mathrm{n}, \mathrm{K}}(\mathrm{s})=\lambda_{\mathrm{c}}(\mathrm{s})+\frac{\lambda_{\mathrm{c}}{ }^{\prime \prime}(\mathrm{s})}{2} \mathrm{~h}_{\mathrm{n}}{ }^{2} \\
& \int_{-1}^{1} \mathrm{z}^{2} \mathrm{~K}(\mathrm{z}) \mathrm{dz}+\mathrm{o}\left(\mathrm{h}_{\mathrm{n}}{ }^{2}\right)
\end{aligned}
$$

For $\mathrm{s}$ is a Lebesgue point from $\lambda_{\mathrm{c}}$.

Proof: From Eq. 7 can be exemplified Eq. 11 and 12:

$$
\begin{aligned}
& Y_{1}=\frac{1}{L_{n, b} h_{n}} \sum_{k=1}^{n_{\varepsilon}} \frac{1}{k_{b}} \int_{0}^{n} K\left(\frac{x-(s+k \tau)}{h_{n}}\right) N(d x) \\
& Y_{2}=\frac{1}{L_{n, b}} \sum_{k=1}^{n_{\varepsilon}} \frac{(s+k \tau)^{b}}{k^{b}}
\end{aligned}
$$

$$
\text { then we get } E \bar{\lambda}_{c, n, k}(s)=E\left(Y_{1}\right)-E\left(Y_{2}\right)
$$

The first term of (12) is Eq. 13:

$$
\begin{aligned}
& E\left(Y_{1}\right)=\frac{1}{L_{n, b} h_{n}} \sum_{k=1}^{n \tau} \frac{1}{k^{b}} \int_{0}^{n} k\left(\frac{x-(s+k \tau)}{h_{n}}\right) E N(d x) \\
& =\frac{1}{L_{n, b} h_{n}} \sum_{k=1}^{n} \frac{1}{k^{b}} \int_{0}^{n} k\left(\frac{x-(s+k \tau)}{h_{n}}\right) \lambda(x)(d x)
\end{aligned}
$$

With the replacement of variables, for example: $y=x$ $(s+k \tau), d y=d x$. Therefore (13) can be written as Eq. 14:

$$
\begin{aligned}
& E\left(Y_{1}\right)=\frac{1}{L_{n, b} h_{n}} \sum_{k=1}^{n \tau} \frac{1}{k^{b}} \int_{R} k\left(\frac{y}{h_{n}}\right) \lambda(y+s+k \tau) I(y+ \\
& s+k \tau \in[0, n]) d y \\
& =\frac{1}{L_{n, b} h_{n}} \sum_{k=1}^{n \tau} \frac{1}{k^{b}} \int_{R} k\left(\frac{y}{h_{n}}\right) \lambda_{c}(y+s) I \\
& (y+s+k \tau \in[0, n]) d y \\
& +\frac{a}{L_{n, b} h_{n}} \sum_{k=1}^{n} \int_{R} k\left(\frac{y}{h_{n}}\right) \frac{(y+s+k \tau)^{b}}{k^{b}} \\
& I(y+s+k \tau \in[0, n]) d y
\end{aligned}
$$

$$
\text { Can be }
$$

noted that $\mathrm{L}_{\mathrm{n}, \mathrm{b}}=\sum_{\mathrm{k}=1}^{\mathrm{n} \tau} \frac{1}{\mathrm{k}^{\mathrm{b}}}=\frac{(\mathrm{n} / \tau)^{1-\mathrm{b}}}{1-\mathrm{b}}+0(1)$ therefore:

$$
\begin{aligned}
& \frac{1}{\mathrm{~L}_{\mathrm{n}, \mathrm{b}}}=\frac{1}{\left((\mathrm{n} / \tau)^{1-\mathrm{b}} /(1-\mathrm{b})+0(1)\right.} \\
& =\frac{1}{\left((\mathrm{n} / \tau)^{1-\mathrm{b}} /(1-\mathrm{b})\right)\left(1+0\left(\frac{1}{\mathrm{n}^{1-\mathrm{b}}}\right)\right)}
\end{aligned}
$$

By using geometric series, above equation can be written as Eq. 15:

$$
\begin{aligned}
& =\frac{1}{\left((\mathrm{n} / \tau)^{1-\mathrm{b}} /(1-\mathrm{b})\right)}\left(1+0\left(\frac{1}{\mathrm{n}^{1-\mathrm{b}}}\right)\right) \\
& =\frac{1-\mathrm{b}}{(\mathrm{n} / \tau)^{1-\mathrm{b}}}+0\left(\frac{1}{\mathrm{n}^{2-2 \mathrm{~b}}}\right)
\end{aligned}
$$

for $n \rightarrow \infty$, then the first term of (14) can be written as Eq. 16:

$$
\begin{aligned}
& \frac{1}{\mathrm{~L}_{\mathrm{n}, \mathrm{h}} \mathrm{h}_{\mathrm{n}}} \sum_{\mathrm{k}=1}^{\mathrm{n} \tau} \frac{1}{\mathrm{k}^{\mathrm{b}}} \int_{\mathrm{R}} \mathrm{k}\left(\frac{\mathrm{y}}{\mathrm{h}_{\mathrm{n}}}\right) \lambda_{\mathrm{c}}(\mathrm{y}+\mathrm{s}) \\
& \mathrm{I}(\mathrm{y}+\mathrm{s}+\mathrm{k} \tau) \in[0, \mathrm{n}]) \mathrm{dy} \\
& =\frac{1}{\mathrm{~h}_{\mathrm{n}}}\left(\frac{1-\mathrm{b}}{(\mathrm{n} / \tau)^{1-\mathrm{b}}}+0\left(\frac{1}{\mathrm{n}^{2-2 b}}\right)\right)\left(\frac{(\mathrm{n} / \tau)^{1-\mathrm{b}}}{1-\mathrm{b}}+0(1)\right)
\end{aligned}
$$


J. Math. \& Stat., 8 (3): 403-412, 2012

$$
\begin{aligned}
& \int_{\mathrm{R}} \mathrm{k}\left(\frac{\mathrm{y}}{\mathrm{h}_{\mathrm{n}}}\right) \lambda_{\mathrm{c}}(\mathrm{y}+\mathrm{s}) \mathrm{dy} \\
& =\frac{1}{\mathrm{~h}_{\mathrm{n}}}\left(1+0\left(\frac{1}{\mathrm{n}^{1-\mathrm{b}}}\right)+0\left(\frac{1}{\mathrm{n}^{1-\mathrm{b}}}\right)\right. \\
& \left.+0\left(\frac{1}{\mathrm{n}^{2-2 b}}\right)\right) \int_{\mathrm{R}} \mathrm{k}\left(\frac{\mathrm{y}}{\mathrm{h}_{\mathrm{n}}}\right) \lambda_{\mathrm{c}}(\mathrm{y}+\mathrm{s}) \mathrm{dy} \\
& =\frac{1}{\mathrm{~h}_{\mathrm{n}}}\left(1+0\left(\frac{1}{\mathrm{n}^{1-b}}\right)\right) \int_{\mathrm{R}} \mathrm{k}\left(\frac{\mathrm{y}}{\mathrm{h}_{\mathrm{n}}}\right) \lambda_{\mathrm{c}}(\mathrm{y}+\mathrm{s}) \mathrm{dy}
\end{aligned}
$$
Eq. 17:

Then using Young's formula we obtained for $n \rightarrow \infty$

$$
\lambda_{\mathrm{c}}(\mathrm{y}+\mathrm{s})=\lambda_{\mathrm{c}}(\mathrm{s})+\lambda_{\mathrm{c}}{ }^{\prime}(\mathrm{s}) \mathrm{y}+\lambda_{\mathrm{c}}{ }^{\prime}(\mathrm{s}) \frac{\mathrm{y}^{2}}{2 !}+\mathrm{o}\left(\mathrm{h}_{\mathrm{n}}^{2}\right)
$$

So we get Eq. 16 as Eq. 18:

$$
=\frac{1}{h_{n}}\left(1+0\left(\frac{1}{n^{1-b}}\right)\right) \int_{R} k\left(\frac{y}{h_{n}}\right)\left(\begin{array}{l}
\lambda_{c}(s)+\lambda_{c}{ }^{\prime}(s) y \\
+\lambda_{c}{ }^{\prime}(s) \frac{y^{2}}{2 !}+o\left(h_{n}^{2}\right)
\end{array}\right) d y
$$

With the replacement of variables, for example $\mathrm{z}=\frac{\mathrm{y}}{\mathrm{h}_{\mathrm{n}}}, \mathrm{dz}=\frac{\mathrm{dy}}{\mathrm{h}_{\mathrm{n}}}$ then (18) can be written as Eq. 19:

$$
\begin{aligned}
& =\frac{1}{\mathrm{~h}_{\mathrm{n}}}\left(1+0\left(\frac{1}{\mathrm{n}^{1-\mathrm{b}}}\right)\right) \int_{\mathrm{R}} \mathrm{k}(\mathrm{z})\left(\begin{array}{c}
\lambda_{\mathrm{c}}(\mathrm{s})+\lambda_{\mathrm{c}}{ }^{\prime}(\mathrm{s}) \mathrm{zh}_{\mathrm{n}} \\
+\lambda_{\mathrm{c}}^{\prime \prime}(\mathrm{s}) \frac{\mathrm{z}^{2} \mathrm{~h}_{\mathrm{n}}^{2}}{2 !}+\mathrm{o}\left(\mathrm{h}_{\mathrm{n}}^{2}\right)
\end{array}\right) \mathrm{dzh} \mathrm{h}_{\mathrm{n}} \\
& =\left(1+0\left(\frac{1}{\mathrm{n}^{1-\mathrm{b}}}\right)\right)\left(\begin{array}{c}
\lambda_{\mathrm{c}}(\mathrm{s}) \int_{\mathrm{R}} \mathrm{k}(\mathrm{z}) \mathrm{dz}+\lambda_{\mathrm{c}}^{\prime}(\mathrm{s}) \mathrm{h}_{\mathrm{n}} \int_{\mathrm{R}} \mathrm{zK}(\mathrm{z}) \mathrm{dz} \\
+\frac{\lambda_{\mathrm{c}}^{\prime \prime}(\mathrm{s})}{2} \mathrm{~h}_{\mathrm{n}}^{2} \int_{\mathrm{R}} \mathrm{z}^{2} \mathrm{~K}(\mathrm{z}) \mathrm{dz}+\mathrm{o}\left(\mathrm{h}_{\mathrm{n}}^{2} \int_{\mathrm{R}} \mathrm{k}(\mathrm{z}) \mathrm{dz}\right)
\end{array}\right) \\
& =\lambda_{\mathrm{c}}(\mathrm{s}) \int_{\mathrm{R}} \mathrm{k}(\mathrm{z}) \mathrm{dz}+\lambda_{\mathrm{c}}^{\prime}(\mathrm{s}) \mathrm{h}_{\mathrm{n}} \int_{\mathrm{R}} \mathrm{zK}(\mathrm{z}) \mathrm{dz} \\
& +\frac{\lambda_{\mathrm{c}}^{\prime \prime}(\mathrm{s})}{2} \mathrm{~h}_{\mathrm{n}}^{2} \int_{\mathrm{R}} \mathrm{z}^{2} \mathrm{k}(\mathrm{z}) \mathrm{dz}+\mathrm{o}\left(\mathrm{h}_{\mathrm{n}}^{2}\right) \int_{\mathrm{R}} \mathrm{k}(\mathrm{z}) \mathrm{dz}+0\left(\frac{1}{\mathrm{n}^{1-b}}\right)
\end{aligned}
$$

Because $\mathrm{K}$ is symmetric and satisfies (K.1) and (K.2) then (19) becomes:

$$
\begin{aligned}
& =\lambda_{\mathrm{c}}(\mathrm{s})+\frac{\lambda_{\mathrm{c}}^{\prime \prime}(\mathrm{s})}{2} \mathrm{~h}_{\mathrm{n}}^{2} \int_{-1}^{1} \mathrm{z}^{2} \mathrm{k}(\mathrm{z}) \mathrm{dz}+\mathrm{o}\left(\mathrm{h}_{\mathrm{n}}^{2}\right) \\
& +0\left(\frac{1}{\mathrm{n}^{1-\mathrm{b}}}\right)=\lambda_{\mathrm{c}}(\mathrm{s})+\frac{\lambda_{\mathrm{c}}^{\prime \prime}(\mathrm{s})}{2} \mathrm{~h}_{\mathrm{n}}^{2} \int_{-1} \mathrm{z}^{2} \mathrm{k}(\mathrm{z}) \mathrm{dz}+\mathrm{o}\left(\mathrm{h}_{\mathrm{n}}^{2}\right) \\
& +0\left(\frac{\mathrm{h}_{\mathrm{n}}^{2}}{\mathrm{n}^{1-\mathrm{b}} \mathrm{h}_{\mathrm{n}}^{2}}\right)
\end{aligned}
$$

For $n \rightarrow \infty$. Because $n^{1-2 b} h_{n}{ }^{2} \rightarrow \infty$, then for $n \rightarrow \infty$ the right side of the above equation becomes Eq. 20: $=\lambda_{\mathrm{c}}(\mathrm{s})+\frac{\lambda_{\mathrm{c}}^{\prime \prime}(\mathrm{s})}{2} \mathrm{~h}_{\mathrm{n}}^{2} \int_{-1}^{1} \mathrm{z}^{2} \mathrm{k}(\mathrm{z}) \mathrm{dz}+\mathrm{o}\left(\mathrm{h}_{\mathrm{n}}^{2}\right)$

The second term of (14) can be described as follows. By using Taylor series, we obtained:

$$
\begin{aligned}
& \frac{(\mathrm{y}+\mathrm{s}+\mathrm{k} \tau)^{\mathrm{b}}}{\mathrm{k}^{\mathrm{b}}}=\left(\tau+\frac{\mathrm{s}}{\mathrm{k}}+\frac{\mathrm{y}}{\mathrm{k}}\right)^{\mathrm{b}}=\left(\tau+\frac{\mathrm{s}}{\mathrm{k}}\right)^{\mathrm{b}} \\
& +\frac{\mathrm{b}}{1 !}\left(\tau+\frac{\mathrm{s}}{\mathrm{k}}\right)+\frac{\mathrm{b}}{1 !}\left(\tau+\frac{\mathrm{s}}{\mathrm{k}}\right)^{\mathrm{b}-1} \frac{\mathrm{y}}{\mathrm{k}}+\frac{\mathrm{b}}{2 !}(\mathrm{b}-1)\left(\tau+\frac{\mathrm{s}}{\mathrm{k}}\right)^{\mathrm{b}-2}\left(\frac{\mathrm{y}}{\mathrm{k}}\right)^{2}+\ldots
\end{aligned}
$$

Because $h_{n} \downarrow 0$ for $n \rightarrow \infty$, then the behavior of $J$ equal to $h_{n}$. So the above equation can be rewritten as:

$$
=\left(\tau+\frac{\mathrm{s}}{\mathrm{k}}\right)^{\mathrm{b}}+\frac{\mathrm{b}}{1 !}\left(\tau+\frac{\mathrm{s}}{\mathrm{k}}\right)^{\mathrm{b}-1} \frac{\mathrm{y}}{\mathrm{k}}+0\left(\frac{\mathrm{h}_{\mathrm{n}}^{2}}{\mathrm{k}^{2}}\right)
$$

For $\mathrm{n} \rightarrow \infty$. So that the second term of (14) becomes:

$$
\begin{aligned}
& \frac{a}{L_{n, b} h_{n}} \sum_{k=1}^{n \tau} \int_{R} k\left(\frac{y}{h_{n}}\right) \frac{(y+s+k \tau)^{b}}{k^{b}} I(y+s+k \tau \in[0, n]) d y \\
& =\frac{a}{L_{n, b} h_{n}} \int_{R} k\left(\frac{y}{h_{n}}\right) \sum_{k=1}^{n}\left(\left(\tau+\frac{s}{k}\right)^{b}+\frac{b}{1 !}\right)\left(\tau+\frac{s}{k}\right)^{b-1} \\
& \left.\frac{y}{k}+0\left(\frac{h_{n}^{2}}{k^{2}}\right)\right) I(y+s+k \tau \in[0, n]) d y
\end{aligned}
$$

With the replacement of variables, $z=\frac{y}{h_{n}}, d z=\frac{d y}{h_{n}}$ the above equation becomes:

$$
\begin{aligned}
& =\frac{\mathrm{a}}{\mathrm{L}_{\mathrm{n} . \mathrm{b}}} \int_{\mathrm{R}} \mathrm{k}(\mathrm{z}) \sum_{\mathrm{k}=1}^{\mathrm{n} \tau}\left(\left(\tau+\frac{\mathrm{s}}{\mathrm{k}}\right)^{\mathrm{b}}+\frac{\mathrm{b}}{1 !}\left(\tau+\frac{\mathrm{s}}{\mathrm{k}}\right)^{\mathrm{b}-1} \frac{\mathrm{zh} \mathrm{n}}{\mathrm{k}}\right) \\
& \left.+0\left(\frac{\mathrm{h}_{\mathrm{n}}^{2}}{\mathrm{k}^{2}}\right)\right) \mathrm{I}\left(\mathrm{zh} \mathrm{h}_{\mathrm{n}}+\mathrm{s}+\mathrm{k} \tau \in[0, \mathrm{n}]\right) \mathrm{dz} \\
& =\frac{\mathrm{a}}{\mathrm{L}_{\mathrm{n}, \mathrm{b}}}\left(\begin{array}{l}
\sum_{\mathrm{k}=1}^{\mathrm{n} \tau}\left(\tau+\frac{\mathrm{s}}{\mathrm{k}}\right)^{\mathrm{b}}+\mathrm{bh} \mathrm{h}_{\mathrm{n}} \sum_{\mathrm{k}=1}^{\mathrm{n} \tau} \frac{1}{\mathrm{k}} \\
\left(\tau+\frac{\mathrm{s}}{\mathrm{k}}\right)^{\mathrm{b}-1} \int_{\mathrm{R}}^{\mathrm{z} K}(\mathrm{z})\left(\mathrm{zh}_{\mathrm{n}}+\mathrm{s}+\mathrm{k} \tau \in[0, \mathrm{n}]\right) \\
\mathrm{dz}+0\left(\mathrm{~h}_{\mathrm{n}}^{2}\right) \sum_{\mathrm{k}=1}^{\mathrm{n} \tau} \frac{1}{\mathrm{k}^{2}}
\end{array}\right)
\end{aligned}
$$

With $\frac{1}{\mathrm{~L}_{\mathrm{n}, \mathrm{b}}}$ as (15), is symmetric and satisfy (K.1) and (K.3) so the above equation can be written as: 


$$
\begin{aligned}
=\left(\frac{a(1-b)}{(n / \tau)^{1-b}}+0\left(\frac{1}{n^{2-2 b}}\right)\right)\left(\sum_{k=1}^{n \tau}\left(\tau+\frac{s}{k}\right)^{b}+0\left(h_{n}^{2}\right) \sum_{k=1}^{n \tau} \frac{1}{k^{2}}\right) \\
=\left(\frac{a(1-b)}{(n / \tau)^{1-b}}+0\left(\frac{1}{n^{2-2 b}}\right)\right) \\
\left.=\left(\sum_{k=1}^{n}\left(\tau+\frac{s}{k}\right)\right)^{b}+0\left(h_{n}^{2}\right) 0(1)\right) \\
=\sum_{k=1}^{n \tau}\left(\tau+\frac{s}{k}\right)^{b}\left(\frac{a(1-b)}{(n / \tau)^{1-b}}+0\left(\frac{1}{n^{2-2 b}}\right)\right) \\
\left.+0\left(h_{n}^{2}\right)\left(\frac{a(1-b)}{(n / \tau)^{1-b}}\right)+0\left(\frac{1}{n^{2-2 b}}\right)\right) \\
=\sum_{k=1}^{n \tau}\left(\tau+\frac{s}{k}\right)^{b}\left(\frac{a(1-b)}{(n / \tau)^{1-b}}+0\left(\frac{1}{n^{2-2 b}}\right)\right) \\
+0\left(\frac{h_{n}^{2}}{n^{1-b}}\right)+0\left(\frac{h_{n}^{2}}{n^{2-2 b}}\right) \\
=\sum_{k=1}^{n \tau}\left(\tau+\frac{s}{k}\right)^{b}\left(\frac{a(1-b)}{(n / \tau)^{1-b}}+0\left(\frac{1}{n^{2-2 b}}\right)\right) \\
+0\left(\frac{h_{n}^{2}}{n^{1-b}}\right)
\end{aligned}
$$

For $\mathrm{n} \rightarrow \infty$. May be noted that, by using Taylor series we obtained:

$$
\begin{aligned}
& \sum_{\mathrm{k}=1}^{\mathrm{n} \tau}\left(\tau+\frac{\mathrm{s}}{\mathrm{k}}\right)^{\mathrm{b}}=\sum_{\mathrm{k}=1}^{\mathrm{n} \tau}\left(\tau^{\mathrm{b}}+0\left(\frac{1}{\mathrm{k}}\right)\right) \\
& =\frac{\mathrm{n}}{\tau} \tau^{\mathrm{b}}+\sum_{\mathrm{k}=1}^{\mathrm{n} \tau} 0\left(\frac{1}{\mathrm{k}}\right)=\frac{\mathrm{n}}{\tau^{1-\mathrm{b}}}+0(1) \sum_{\mathrm{k}=1}^{\mathrm{n} \tau} \frac{1}{\mathrm{k}} \\
& =\frac{\mathrm{n}}{\tau^{1-\mathrm{b}}}+0(1)
\end{aligned}
$$

So the above equation can be written as Eq. 21:

$$
\begin{aligned}
& =\left(\frac{\mathrm{n}}{\tau^{1-\mathrm{b}}}+0(1)\right)\left(\frac{\mathrm{a}(1-\mathrm{b})}{(\mathrm{n} / \tau)^{1-b}}+0\left(\frac{1}{\mathrm{n}^{2-2 \mathrm{~b}}}\right)\right)+0\left(\frac{\mathrm{h}_{\mathrm{n}}^{2}}{\mathrm{n}^{1-\mathrm{b}}}\right) \\
& =\mathrm{a}(1-\mathrm{b}) \mathrm{n}^{\mathrm{b}}+0\left(\frac{1}{\mathrm{n}^{1-2 b}}\right)+0\left(\frac{1}{\mathrm{n}^{1-\mathrm{b}}}\right) \\
& +0\left(\frac{1}{\mathrm{n}^{2-2 \mathrm{~b}}}\right)+0\left(\frac{\mathrm{h}_{\mathrm{n}}^{2}}{\mathrm{n}^{1-\mathrm{b}}}\right)= \\
& \mathrm{a}(1-\mathrm{b}) \mathrm{n}^{\mathrm{b}}+0\left(\frac{1}{\mathrm{n}^{1-2 \mathrm{~b}}}\right) 0\left(\frac{\mathrm{h}_{\mathrm{n}}^{2}}{\mathrm{n}^{1-\mathrm{b}}}\right)
\end{aligned}
$$

The second term of (12) is deterministic, then we obtained Eq. 22:

$$
\begin{aligned}
& E\left(Y_{2}\right)=E\left(\frac{a}{L_{n, b}} \sum_{k=1}^{n \tau} \frac{(s+k \tau)}{k^{b}}\right) \\
& =\frac{a}{L_{n, b}} \sum_{k=1}^{n \tau} \frac{(s+k \tau)}{k^{b}} \\
& \text { With } \frac{1}{L_{n . b}} \text { as (15), right side of Eq. } 22 \text { becomes }
\end{aligned}
$$

Eq. 23:

$$
\begin{aligned}
& =\left(\frac{a(1-b)}{(n / \tau)^{1-b}}+0\left(\frac{1}{n^{2-2 b}}\right)\right) \sum_{k=1}^{n \tau}\left(\tau+\frac{s}{k}\right)^{b} \\
& =\left(\frac{a(1-b)}{(n / \tau)^{1-b}}+0\left(\frac{1}{n^{2-2 b}}\right)\right)\left(\frac{n}{\tau^{1-b}}+0(1)\right) \\
& =a(1-b) n^{b}+0\left(\frac{1}{n^{1-b}}\right)+0\left(\frac{1}{n^{1=2 b}}\right)+0\left(\frac{1}{n^{2-2 n}}\right) \\
& =a(1-b) n^{b}+0\left(\frac{1}{n^{1-b}}\right)+0\left(\frac{1}{n^{1-2 b}}\right)+0\left(\frac{1}{n^{2-2 b}}\right) \\
& =a(1-b) n^{b} 0\left(\frac{1}{n^{1-2 b}}\right)
\end{aligned}
$$

By combining (20), (21) and (23) we obtained:

$$
\begin{aligned}
& E \bar{\lambda}_{c, n, k}(s) \\
& =\lambda_{c}(s)+\frac{\lambda_{c}^{\prime \prime}(s)}{2} h_{n}^{2} \int_{-1}^{1} z^{2} k(z) d z+o\left(h_{n}^{2}\right)+a \\
& (1-b) n^{b}+0\left(\frac{1}{n^{1-2 b}}\right)+0\left(\frac{1}{n^{1-2 b}}\right)+\left(\frac{h_{n}^{2}}{n^{1-b}}\right) \\
& -\left(a(1-b) n^{b}+0\left(\frac{1}{n^{1-2 b}}\right)\right) \\
& =\lambda_{c}(s)+\frac{\lambda_{c}^{\prime \prime}(s)}{2} h_{n}^{2} \int_{-1}^{1} z^{2} k(z) d z+0\left(h_{n}^{2}\right) \\
& +0\left(\frac{1}{n^{1-2 b}}\right)+0\left(\frac{h_{n}^{2}}{n^{1-b}}\right) \\
& =\lambda_{c}(s)+\frac{\lambda_{c}^{\prime \prime}(s)}{2} h_{n}^{2} \int_{-1}^{1} z^{2} k(z) d z+o\left(h_{n}^{2}\right) \\
& +0\left(\frac{h_{n}^{2}}{n^{1-b} h_{n}^{2}}\right)+0\left(h_{n}^{2}\right) \frac{1}{n^{1-b}}
\end{aligned}
$$

For $\mathrm{n} \rightarrow \infty$ Because $\frac{1}{\mathrm{n}^{1-b}} \rightarrow 0$ dan $\mathrm{n}^{1-2 b} \mathrm{~h}_{\mathrm{n}}{ }^{2} \rightarrow \infty$ for $\mathrm{n} \rightarrow \infty$, then the fourth and fifth term of the above equation is o $\left(\mathrm{h}_{\mathrm{n}}{ }^{2}\right)$. So that the above equation can be written as Eq. 24:

$$
=\lambda_{\mathrm{c}}(\mathrm{s})+\frac{\lambda_{\mathrm{c}}^{\prime \prime}(\mathrm{s})}{2} \mathrm{~h}_{\mathrm{n}}^{2} \int_{-1}^{1} \mathrm{z}^{2} \mathrm{k}(\mathrm{z}) \mathrm{dz}+\mathrm{o}\left(\mathrm{h}_{\mathrm{n}}^{2}\right)
$$


For $\mathrm{n} \rightarrow \mathrm{a}$. Theorem 1 is proven.

Theorem 2: Asymptotic approximation of the variance for the estimator: Suppose the intensity function $\lambda$ as (1) and locally integrable. If kernel $\mathrm{k}$ satisfies (K.1), (K.2), (K.3), $h_{n} \downarrow 0, n^{1-b} h_{n} \rightarrow \infty$ for $n \rightarrow$ $\infty$ and $\lambda_{\mathrm{c}}$ bounded around $s$, then for $\mathrm{n} \rightarrow \infty$ Eq. 25:

$$
\operatorname{var} \bar{\lambda}_{c, n, k}(s)=\frac{a(1-b) \tau^{b}}{(n / \tau)^{1-b} h_{n}} \int_{-1}^{1} k^{2}(z) d z+o\left(\frac{1}{n^{1-b} h_{n}}\right)
$$

Proof: From Eq. 11 and because $\mathrm{Y}_{2}$ is deterministic, then Eq. 26:

$$
\operatorname{Var} \bar{\lambda}_{\mathrm{c}, \mathrm{n}, \mathrm{k}}(\mathrm{s})=\operatorname{Var}\left(\mathrm{Y}_{1}\right)
$$

For large value of $\mathrm{n}$ and $\mathrm{k} \neq \mathrm{j}$, the interval $[\mathrm{s}+\mathrm{k} \tau$ $\left.h_{n}, s+k \tau+h_{n}\right]$ and $\left[s+j \tau-h_{n}, s+j \tau+h_{n}\right]$ do not overlap so that for all $\quad k \neq j, k\left(\frac{x-(s+k \tau)}{h_{n}}\right) N(d x)$ and $\mathrm{k}\left(\frac{\mathrm{x}-(\mathrm{s}+\mathrm{j} \tau)}{\mathrm{h}_{\mathrm{n}}}\right) \mathrm{N}(\mathrm{dx})$ are independent. So that $\operatorname{Var}\left(\mathrm{Y}_{1}\right)$ can be determined as follows Eq. 27:

$$
\begin{aligned}
& \operatorname{Var}\left(Y_{1}\right) \\
& =\operatorname{Var}\left(\frac{1}{L_{n, b} h_{n}} \sum_{k=1}^{n} \frac{1}{k^{b}} \int_{0}^{n} k\left(\frac{x-(s+k \tau)}{h_{n}}\right) N(d x)\right) \\
& =\frac{1}{\left(L_{n, b} h_{n}\right)^{2}} \sum_{k=1}^{n \tau} \frac{1}{k^{2 b}} \int_{0}^{n} k^{2}\left(\frac{x-(s+k \tau)}{h_{n}}\right) \operatorname{var}(N(d x))
\end{aligned}
$$

Because $\mathrm{N}$ is a Poisson process, then $\operatorname{Var}(\mathrm{N})=\mathrm{E}$ (N) so that (27) becomes Eq. 28:

$$
\begin{aligned}
& =\frac{1}{\left(L_{n, b} h_{n}\right)^{2}} \sum_{k=1}^{n \tau} \frac{1}{k^{2 b}} \int_{0}^{n} k^{2}\left(\frac{x-(s+k \tau)}{h_{n}}\right) E(N(d x)) \\
& =\frac{1}{\left(L_{n, b} h_{n}\right)^{2}} \sum_{k=1}^{n \tau} \frac{1}{k^{2 b}} \int_{0}^{n} k^{2}\left(\frac{x-(s+k \tau)}{h_{n}}\right) \lambda(x)
\end{aligned}
$$

With the replacement of variables, $y=x-(s+k \tau)$ $d y=d x$, then (28) can be written as Eq. 29:

$$
\begin{aligned}
& \operatorname{Var}\left(Y_{1}\right)=\frac{1}{\left(L_{n, b} h_{n}\right)^{2}} \sum_{k=1}^{n \tau} \frac{1}{k^{2 b}} \int_{R} k^{2}\left(\frac{y}{h_{n}}\right) \\
& \lambda(y+s+k \tau) I(y+s+k \tau \in[0, n]) d y
\end{aligned}
$$

By combining (1) and (2), the right side of (29) becomes Eq. 30:

$$
\begin{aligned}
& \frac{1}{\left(L_{n, b} h_{n}\right)^{2}} \sum_{k=1}^{n \tau} \frac{1}{k^{2 b}} \int_{R} k^{2}\left(\frac{y}{h_{n}}\right)\left(\lambda_{c}(y+s)\right) \\
& \left.\left.+a(y+s+k \tau)^{b}\right) I(y+s+k \tau) \in[0, n]\right) d y \\
& =\frac{1}{\left(L_{n, b} h_{n}\right)^{2}} \sum_{K=1}^{n \tau} \frac{1}{k^{2 b}} \int_{R} k^{2}\left(\frac{y}{h_{n}}\right) \lambda_{c}(y+s) I(y+s) \\
& +k \tau \in[0, n)] d y+\frac{a}{\left(L_{n, b} h_{n}\right)^{2}} \sum_{k=1}^{n \tau} \frac{1}{k^{2 b}} \int_{R} k^{2}\left(\frac{y}{h_{n}}\right) \\
& (y+s+k \tau)^{b} I(y+s+k \tau \in[0, n]) d y
\end{aligned}
$$

Because $\lambda_{\mathrm{c}}$ is bounded around $\mathrm{s}$ then $\lambda_{\mathrm{c}}(\mathrm{y}+\mathrm{s}) \leq$ $\lambda_{0}, \lambda_{0}$ is a constant value, so that the first term of the right side of Eq. 30 can be written as Eq. 31:

$$
\begin{aligned}
& \frac{1}{\left(L_{n, b} h_{n}\right)^{2}} \sum_{k=1}^{n \tau} \frac{1}{k^{2 b}} \int_{R} k^{2}\left(\frac{y}{h_{n}}\right) \\
& \left.\lambda_{c}(y+s) I(y+s+k \tau) \in[0, n]\right) d y \\
& \leq \frac{1}{\left(L_{n, b} h_{n}\right)^{2}} \sum_{k=1}^{n \tau} \frac{1}{k^{2 b}} \int_{R} k^{2}\left(\frac{y}{h_{n}}\right) \lambda_{0} I \\
& (y+s+k \tau \in[0, n]) d y
\end{aligned}
$$

With the replacement of variables, $\mathrm{z}=\frac{\mathrm{y}}{\mathrm{h}_{\mathrm{n}}}, \mathrm{dz}=\frac{\mathrm{dy}}{\mathrm{h}_{\mathrm{n}}}$ the right side of (31) becomes Eq. 32:

$=\frac{\lambda_{0}}{\mathrm{~L}_{\mathrm{n}, \mathrm{b}}{ }^{2} \mathrm{~h}_{\mathrm{n}}} \sum_{\mathrm{k}=1}^{\mathrm{n} \tau} \frac{1}{\mathrm{k}^{2 \mathrm{~b}}} \int_{\mathrm{R}} \mathrm{k}^{2}(\mathrm{z}) \mathrm{I}(\mathrm{zh} \mathrm{n}+\mathrm{s}+\mathrm{k} \tau)$

$\in[0, \mathrm{n}]) \mathrm{dz}$

Because K satisfies (K.3) then (32) can be written as Eq. 33:

$$
\begin{aligned}
& =\frac{\lambda_{0}}{\mathrm{~L}_{\mathrm{n}, \mathrm{b}}{ }^{2} \mathrm{~h}_{\mathrm{n}}} \int_{-1}^{1} \mathrm{k}^{2}(\mathrm{z}) \mathrm{dz} \sum_{\mathrm{k}=1}^{\mathrm{n} \tau} \frac{1}{\mathrm{k}^{2 \mathrm{~b}}} \mathrm{I}\left(\mathrm{zh} \mathrm{n}_{\mathrm{n}}+\mathrm{s}+\mathrm{k} \tau\right) \\
& \in[0, \mathrm{n}])
\end{aligned}
$$

To determine the quantity of the right side of (33), we divided in three cases, for $0<\mathrm{b}<\frac{1}{2}, \mathrm{~b}=\frac{1}{2}$ and $\frac{1}{2}<\mathrm{b}<1$ For $0<\mathrm{b}<\frac{1}{2}$ can be noted that Eq. 34:

$\sum_{\mathrm{k}=1}^{\mathrm{n} \tau} \frac{1}{\mathrm{k}^{2 b}} \mathrm{I}(\mathrm{zh}+\mathrm{n}+\mathrm{k} \tau \in[0, \mathrm{n}])$

$$
=\frac{\left(\frac{\mathrm{n}}{\tau}\right)^{1-2 \mathrm{~b}}}{1-2 \mathrm{~b}}+0
$$


For $\mathrm{n} \rightarrow$. By using the result that we obtained from (15) and (34), then the right side of (33) becomes:

$$
\begin{aligned}
& \frac{\lambda_{0}}{\mathrm{~L}_{\mathrm{n}, \mathrm{b}}{ }^{2} \mathrm{~h}_{\mathrm{n}}} \int_{-1}^{1} \mathrm{k}^{2}(\mathrm{z}) \mathrm{dz} \sum_{\mathrm{k}=1}^{\mathrm{n} \tau} \frac{1}{\mathrm{k}^{2 b}} \mathrm{I}\left(\mathrm{zh} \mathrm{h}_{\mathrm{n}}+\mathrm{s}+\mathrm{k} \tau \in[0, \mathrm{n}]\right) \\
& =\frac{\lambda_{0}}{\mathrm{~h}_{\mathrm{n}}} \int_{-1}^{1} \mathrm{k}^{2}(\mathrm{z}) \mathrm{dz}\left(\frac{1-\mathrm{b}}{(\mathrm{n} / \tau)^{1-\mathrm{b}}}+0\left(\frac{1}{\mathrm{n}^{2-2 \mathrm{~b}}}\right)^{2}\right) \\
& \left(\frac{(\mathrm{n} / \tau)^{1-2 \mathrm{~b}}}{1-2 \mathrm{~b}}+0(1)\right) \\
& =\frac{\lambda_{0}}{\mathrm{~h}_{\mathrm{n}}} \int_{-1}^{1} \mathrm{k}^{2}(\mathrm{z}) \mathrm{dz}\left(\frac{(1-\mathrm{b})^{2}}{(\mathrm{n} / \tau)^{2-2 \mathrm{~b}}}+0\left(\frac{1}{\mathrm{n}^{3-3 \mathrm{~b}}}\right)\right. \\
& \left.+0\left(\frac{1}{n^{4-4 b}}\right)\right)\left(\frac{(\mathrm{n} / \tau)^{1-2 b}}{1-2 b}+0(1)\right) \\
& =\frac{\lambda_{0}}{\mathrm{~h}_{\mathrm{n}}} \int_{-1}^{1} \mathrm{k}^{2}(\mathrm{z}) \mathrm{dz}\left(\frac{(1-\mathrm{b})^{2}}{(\mathrm{n} / \tau)^{2-2 b}}+0\left(\frac{1}{\mathrm{n}^{3-3 b}}\right)\right) \\
& \left(\frac{(\mathrm{n} / \tau)^{1-2 b}}{1-2 \mathrm{~b}}+0(1)\right) \\
& =\lambda_{0} \int_{-1}^{1} k^{2}(z) d z\left(\begin{array}{l}
\frac{(1-b)^{2}}{(1-2 b) n h_{n}}+0\left(\frac{1}{n^{2-2 b} h_{n}}\right) \\
+0\left(\frac{1}{n^{2-b} h_{n}}\right)+0\left(\frac{1}{n^{3-3 b} h_{n}}\right)
\end{array}\right)
\end{aligned}
$$

By taking the largest quantity, the above equation can be written as Eq. 35:

$$
=\frac{(1-b)^{2} \lambda_{0}}{(1-2 b) n h_{n}} \int_{-1}^{1} k^{2}(z) d z+0\left(\frac{1}{n^{2-2 b} h_{n}}\right)
$$

For $\mathrm{n} \rightarrow \infty$. For $\mathrm{b}=\frac{1}{2}$, can be noted that Eq. 36:

$\sum_{\mathrm{k}=1}^{\mathrm{n} \tau} \frac{1}{\mathrm{k}^{2 \mathrm{~b}}}\left(\mathrm{zh}_{\mathrm{n}}+\mathrm{s}+\mathrm{k} \tau \in[0, \mathrm{n}]\right)=\operatorname{In} \mathrm{n}+0(1)$

For $\mathrm{n} \rightarrow \infty$. By using the result that we obtained from (15) and (36), the right side quantity of (33) becomes:

$$
\begin{aligned}
& \frac{\lambda_{0}}{\mathrm{~L}_{\mathrm{n}, \mathrm{b}}{ }^{2} \mathrm{~h}_{\mathrm{n}}} \int_{-1}^{1} \mathrm{k}^{2}(\mathrm{z}) \mathrm{dz} \sum_{\mathrm{k}=1}^{\mathrm{n} \tau} \frac{1}{\mathrm{k}^{2 \mathrm{~b}}} \mathrm{I}\left(\mathrm{zh}_{\mathrm{n}}+\mathrm{s}+\mathrm{k} \tau \in[0, \mathrm{n}]\right) \\
& =\frac{\lambda_{0}}{\mathrm{~h}_{\mathrm{n}}} \int_{-1}^{1} \mathrm{k}^{2}(\mathrm{z}) \mathrm{dz}\left(\frac{1-\mathrm{b}}{(\mathrm{n} / \tau)^{1-\mathrm{b}}}+0\left(\frac{1}{\mathrm{n}^{2-2 \mathrm{~b}}}\right)\right)^{2}(\mathrm{In} \mathrm{n}+0(1))
\end{aligned}
$$

$$
\begin{aligned}
& =\frac{\lambda_{0}}{\mathrm{~h}_{\mathrm{n}}} \int_{-1}^{1} \mathrm{k}^{2}(\mathrm{z}) \mathrm{dz}\left(\frac{(1-\mathrm{b})^{2}}{(\mathrm{n} / \tau)^{2-2 b}}+0\left(\frac{1}{\mathrm{n}^{3-3 b}}\right)\right) \\
& \text { In } \mathrm{n}+0(1))=\lambda_{0} \int_{-1}^{1} \mathrm{k}^{2}(\mathrm{z}) \mathrm{dz}\left(\frac{(1-\mathrm{b})^{2}}{(\mathrm{n} / \tau)^{2-2 b} \mathrm{~h}_{\mathrm{n}}}+0\right) \\
& \left.\left(\frac{1}{\mathrm{n}^{2-2 b} \mathrm{~h}_{\mathrm{n}}}\right)+0\left(\frac{1}{\left(\mathrm{n}^{3-3 b} / \operatorname{Inn}\right) \mathrm{h}_{\mathrm{n}}}\right)+0\left(\frac{1}{\mathrm{n}^{3-3 b} \mathrm{~h}_{\mathrm{n}}}\right)\right) \\
& \quad=\frac{(1-\mathrm{b})^{2} \lambda_{0} \operatorname{In~n}}{(\mathrm{n} / \tau)^{2-2 b} \mathrm{~h}_{\mathrm{n}}} \int_{-1}^{1} \mathrm{k}^{2}(\mathrm{z}) \mathrm{dz}+0\left(\frac{1}{\mathrm{n}^{2-2 b} \mathrm{~h}_{\mathrm{n}}}\right) \\
& \quad+0\left(\frac{1}{\left(\mathrm{n}^{3-3 b} / \operatorname{Inn}\right) \mathrm{h}_{\mathrm{n}}}\right)+0\left(\frac{1}{\mathrm{n}^{3-3 b} \mathrm{~h}_{\mathrm{n}}}\right)
\end{aligned}
$$

By looking the largest quantity, the above equation becomes Eq. 37:

$$
\begin{aligned}
& =\frac{(1-b)^{2} \lambda_{0} \operatorname{Inn} n}{(n / \tau)^{2-2 b} h_{n}} \int_{-1}^{1} k^{2}(z) d z+ \\
& 0\left(\frac{1}{n^{2-2 b} h_{n}}\right)
\end{aligned}
$$

For $\mathrm{n} \rightarrow \infty$ For $\frac{1}{2}<\mathrm{b}<1$ can be noted that Eq. 38:

$\sum_{\mathrm{k}=1}^{\mathrm{n} \tau} \frac{1}{\mathrm{k}^{2 \mathrm{~b}}} \mathrm{I}\left(\mathrm{zh}_{\mathrm{n}}+\mathrm{s}+\mathrm{k} \tau \in[0, \mathrm{n}]\right)=0(1)$

For $n \rightarrow \infty$ So that the right side of (33) can be written as Eq. 39:

$$
\begin{aligned}
& \frac{\lambda_{0}}{\mathrm{~L}_{\mathrm{n}, \mathrm{b}}{ }^{2} \mathrm{~h}_{\mathrm{n}}} \int_{-1}^{1} \mathrm{k}^{2}(\mathrm{z}) \mathrm{dz} \sum_{\mathrm{k}=1}^{\mathrm{n} \tau} \frac{1}{\mathrm{k}^{2 \mathrm{~b}}} \mathrm{I}\left(\mathrm{zh}_{\mathrm{n}}+\mathrm{s}+\mathrm{k} \tau \in[0, \mathrm{n}]\right) \\
& =\frac{\lambda_{0}}{\mathrm{~h}_{\mathrm{n}}} \int_{-1}^{1} \mathrm{k}^{2}(\mathrm{z}) \mathrm{dz}\left(\frac{1-\mathrm{b}}{(\mathrm{n} / \tau)^{1-\mathrm{b}}}+0\left(\frac{1}{\mathrm{n}^{2-2 b}}\right)\right) 0(1) \\
& =\frac{\lambda_{0}}{\mathrm{~h}_{\mathrm{n}}} \int_{-1}^{1} \mathrm{k}^{2}(\mathrm{z}) \mathrm{dz}\left(\frac{(1-\mathrm{b})^{2}}{(\mathrm{n} / \tau)^{2-2 b}}+0\left(\frac{1}{\mathrm{n}^{3-3 b}}\right)\right) 0(1) \\
& =\lambda_{0} \int_{-1}^{1} \mathrm{k}^{2}(\mathrm{z}) \mathrm{dz}\left(0\left(\frac{1}{\mathrm{n}^{2-2 b} \mathrm{~h}_{\mathrm{n}}}\right)+0\left(\frac{1}{\mathrm{n}^{3-3 b} \mathrm{~h}_{\mathrm{n}}}\right)\right) \\
& =0\left(\frac{1}{\mathrm{n}^{2-2 b} \mathrm{~h}_{\mathrm{n}}}\right)+0\left(\frac{1}{\mathrm{n}^{3-3 b} \mathrm{~h}_{\mathrm{n}}}\right) \\
& =0\left(\frac{1}{\mathrm{n}^{2-2 b} \mathrm{~h}_{\mathrm{n}}}\right)
\end{aligned}
$$

For $n \rightarrow \infty$ By taking the largest quantity of (35), (37) and (39) can be obtained the quantity of (33) which also states the quantity of the first term of (30), the quantity is Eq. 40 : 
$0\left(\frac{1}{n^{2-2 b} h_{n}}\right)$

For $\mathrm{n} \rightarrow \infty$ Furthermore, it can be considered the second term of the right side of (30). By using Taylor series expansion, we obtained:

$$
\begin{aligned}
& (y+s+k \tau)^{b} \\
& =(s+k \tau)^{b}+b(s+k \tau)^{b-1} \frac{y}{1 !} \\
& +b(b-1)(s+k \tau)^{b-2} \frac{y^{2}}{2 !}+\ldots
\end{aligned}
$$

Because $h_{n} \downarrow 0$ for $n \rightarrow \infty$ a then the behavior of $y$ is equal to $h_{n}$. So the above equation can be written as Eq. 41:

$$
=(\mathrm{s}+\mathrm{k} \tau)^{\mathrm{b}}+0\left(\mathrm{~h}_{\mathrm{n}}\right)
$$

By using (41), the second term of (30) can be written as Eq. 42:

$\frac{\mathrm{a}}{\left(\mathrm{L}_{\mathrm{n}, \mathrm{b}} \mathrm{h}_{\mathrm{n}}\right)^{2}} \sum_{\mathrm{k}=1}^{\mathrm{n} \tau} \frac{1}{\mathrm{k}^{2 \mathrm{~b}}} \int_{\mathrm{R}} \mathrm{k}^{2}\left(\frac{\mathrm{y}}{\mathrm{h}_{\mathrm{n}}}\right)(\mathrm{y}+\mathrm{s}+\mathrm{k} \tau)^{\mathrm{b}}$

$\mathrm{I}(\mathrm{y}+\mathrm{s}+\mathrm{k} \tau \in[0, \mathrm{n}]) \mathrm{dy}$

$=\frac{\mathrm{a}}{\left(\mathrm{L}_{\mathrm{n}, \mathrm{b}} \mathrm{h}_{\mathrm{n}}\right)^{2}} \sum_{\mathrm{k}=1}^{\mathrm{n}} \frac{1}{\mathrm{k}_{2 \mathrm{~b}}} \int_{\mathrm{R}} \mathrm{k}^{2}\left(\frac{\mathrm{y}}{\mathrm{h}_{\mathrm{n}}}\right)\left((\mathrm{s}+\mathrm{k} \tau)^{\mathrm{b}}\right.$

$\left.+0\left(h_{n}\right)\right) I(y+s+k \tau \in[0, n]) d y$

With the replacement of variables $z=\frac{y}{h_{n}}, d z=\frac{d y}{h_{n}}$ and because k satisfies (K.3) then (42) can be written as:

$$
\begin{aligned}
& =\frac{\mathrm{a}}{1_{\mathrm{n}, \mathrm{b}}{ }^{2} \mathrm{~h}_{\mathrm{n}}} \sum_{\mathrm{k}=1}^{\mathrm{n} \tau} \frac{1}{\mathrm{k}^{2 \mathrm{~b}}} \int_{-1}^{1} \mathrm{k}^{2}(\mathrm{z})\left((\mathrm{s}+\mathrm{k} \tau)^{\mathrm{b}}\right. \\
& \left.\left.+0\left(\mathrm{~h}_{\mathrm{n}}\right)\right) \mathrm{I}\left(\mathrm{zh} \mathrm{h}_{\mathrm{n}}+\mathrm{s}+\mathrm{k} \tau\right) \in[0, \mathrm{n}]\right) \mathrm{dz}
\end{aligned}
$$
Eq. 43:

Using (15) the above equation can be written as

$$
\begin{aligned}
& =\frac{a}{h_{n}}\left(\frac{1}{(n / \tau)^{1-b}}+0\left(\frac{1}{n^{2-2 b}}\right)\right)^{2} \\
& \sum_{k=1}^{n} \frac{1}{k^{2 b}} \int_{-1}^{1} k^{2}(z) d z\left((s+k \tau)^{b}\right. \\
& \left.+0\left(h_{n}\right)\right) I\left(z h_{n}+s+k \tau \in[0, n]\right) \\
& \left.=\frac{a}{h_{n}}\left(\frac{(1-b)^{2}}{(n / \tau)^{2-2 b}}\right)+0\left(\frac{1}{n^{3-3 b}}\right)\right) \int_{-1}^{1} k^{2}(z) d z
\end{aligned}
$$

$$
\sum_{\mathrm{k}=1}^{\mathrm{n} \tau} \frac{(\mathrm{s}+\mathrm{k} \tau)}{\mathrm{k}^{2 \mathrm{~b}}} \mathrm{I}\left(\mathrm{zh}_{\mathrm{n}}+\mathrm{s}+\mathrm{k} \tau \in[0, \mathrm{n}]\right)
$$$$
+\frac{a}{h_{n}}\left(\frac{(1-b)^{2}}{\left(\frac{n}{\tau}\right)^{2-2 b}}+0\left(\frac{1}{n^{3-3 b}}\right)\right)
$$

$$
\left(\int_{-1}^{1} \mathrm{k}^{2} \mathrm{dz} \sum_{\mathrm{k}=1}^{\mathrm{n} \tau} \frac{1}{\mathrm{k}^{2 \mathrm{~b}}} \mathrm{I}\left(\mathrm{zh} \mathrm{h}_{\mathrm{n}}+\mathrm{s}+\mathrm{k} \tau \in[0, \mathrm{n}]\right)\right) O\left(\mathrm{~h}_{\mathrm{n}}\right)
$$

In the same way, by looking at each case the Eq. 43 becomes Eq. 44:

$$
\begin{aligned}
& =\frac{a}{h_{n}}\left(\frac{(1-b)^{2}}{\left(\frac{n}{\tau}\right)^{2-2 b}}+0\left(\frac{1}{n^{3-3 b}}\right)\right) \int_{-1}^{1} k^{2}(z) d z \\
& \sum_{k=1}^{n \tau} \frac{(s+k \tau)^{b}}{k^{2 b}} I\left(z h_{n}+s+k \tau \in[0, n]\right)+0\left(\frac{1}{n^{2-2 b} h_{n}}\right)
\end{aligned}
$$

For $\mathrm{n} \rightarrow \infty$ Can be noted that Eq. 45:

$$
\begin{aligned}
& \sum_{\mathrm{k}=1}^{\mathrm{n} \tau} \frac{(\mathrm{s}+\mathrm{k} \tau)^{\mathrm{b}}}{\mathrm{k}^{2 \mathrm{~b}}} \mathrm{I}\left(\mathrm{zh} \mathrm{h}_{\mathrm{n}}+\mathrm{s}+\mathrm{k} \tau \in[0, \mathrm{n}]\right) \\
& =\sum_{\mathrm{k}=1}^{\mathrm{n} \tau}\left(\frac{\mathrm{s}}{\mathrm{k}^{2}}+\frac{\tau}{\mathrm{k}}\right) \mathrm{I}\left(\mathrm{zh}_{\mathrm{n}}+\mathrm{s}+\mathrm{k} \tau \in[0, \mathrm{n}]\right)
\end{aligned}
$$

By using Taylor series expansion we obtained:

$$
\begin{aligned}
& \left(\frac{\mathrm{s}}{\mathrm{k}^{2}}+\frac{\tau}{\mathrm{k}}\right)^{\mathrm{b}}=\left(\frac{\tau}{\mathrm{k}}\right)^{\mathrm{b}}+\frac{\mathrm{b}}{1 !}\left(\frac{\tau}{\mathrm{k}}\right)^{\mathrm{b}-1} \frac{\mathrm{s}}{\mathrm{k}^{2}}+\frac{\mathrm{b}}{2 !} \\
& (\mathrm{b}-1)\left(\frac{\tau}{\mathrm{k}}\right)^{\mathrm{b}-2}\left(\frac{\mathrm{s}}{\mathrm{k}^{2}}\right)^{2}
\end{aligned}
$$

Because $\frac{\mathrm{s}}{\mathrm{k}^{2}} \rightarrow 0$ for $\mathrm{n} \rightarrow \infty$, then the above equation becomes:

$$
\begin{aligned}
& \left(\frac{\mathrm{s}}{\mathrm{k}^{2}}+\frac{\tau}{\mathrm{k}}\right)^{\mathrm{b}}=\left(\frac{\tau}{\mathrm{k}}\right)^{\mathrm{b}}+\mathrm{o}\left(\frac{1}{\mathrm{k}^{\mathrm{b}-1}} \frac{1}{\mathrm{k}^{2}}\right) \\
& =\left(\frac{\tau}{\mathrm{k}}\right)^{\mathrm{b}}+0\left(\frac{1}{\mathrm{k}^{\mathrm{b}+1}}\right)
\end{aligned}
$$

So that, Eq. 45 becomes:

$$
\begin{aligned}
& \sum_{k=1}^{n \tau}\left(\frac{s}{k^{2}}+\frac{\tau}{k}\right)^{b} I\left(\mathrm{zh}_{\mathrm{n}}+\mathrm{s}+\mathrm{k} \tau \in[0, \mathrm{n}]\right) \\
& =\sum_{\mathrm{k}=1}^{\mathrm{n} \tau}\left(\left(\frac{\tau}{\mathrm{k}}\right)^{\mathrm{b}}+0\left(\frac{1}{\mathrm{k}^{\mathrm{b}+1}}\right)\right)
\end{aligned}
$$




$$
\begin{aligned}
& =\sum_{\mathrm{k}=1}^{\mathrm{n} \tau}\left(\frac{\tau}{\mathrm{k}}\right)^{\mathrm{b}}+\sum_{\mathrm{k}=1}^{\mathrm{n} \tau} 0\left(\frac{1}{\mathrm{k}^{\mathrm{b}+1}}\right) \\
& =\tau^{\mathrm{b}} \sum_{\mathrm{k}=1}^{\mathrm{n} \tau} \frac{1}{\mathrm{k}^{\mathrm{b}}}+0(1) \sum_{\mathrm{k}=1}^{\mathrm{n} \tau} \frac{1}{\mathrm{k}^{\mathrm{b}+1}} \\
& =\tau^{\mathrm{b}} \sum_{\mathrm{k}=1}^{\mathrm{n} \tau} \frac{1}{\mathrm{k}^{\mathrm{b}}}+0(1)
\end{aligned}
$$

We may be recalled that 3 $\mathrm{L}_{\mathrm{n}, \mathrm{b}}=\sum_{\mathrm{k}=1}^{\mathrm{n} \tau} \frac{1}{\mathrm{k}^{\mathrm{b}}}=\frac{(\mathrm{n} / \tau)^{1-\mathrm{b}}}{1-\mathrm{b}}+0(1)$ so the above equation can be written as Eq. 46:

$$
\begin{aligned}
& =\tau^{\mathrm{b}}\left(\frac{(\mathrm{n} / \tau)^{1-\mathrm{b}}}{1-\mathrm{b}}+0(1)\right)+0(1) \\
& =\frac{(\mathrm{n} / \tau)^{1-\mathrm{b}} \tau^{\mathrm{b}}}{1-\mathrm{b}}+0(1)
\end{aligned}
$$

For $n \rightarrow \infty$ By combining (46) into (44) we can obtained Eq. 47:

$$
\begin{aligned}
& =\frac{\mathrm{a}}{\mathrm{h}_{\mathrm{n}}}\left(\frac{(1-\mathrm{b})^{2}}{(\mathrm{n} / \mathrm{t})^{2-2 \mathrm{~b}}}+0\left(\frac{1}{\mathrm{n}^{3-3 \mathrm{~b}}}\right)\right) \int_{-1}^{1} \mathrm{k}^{2}(\mathrm{z}) \mathrm{dz} \\
& \left(\frac{(\mathrm{n} / \tau)^{1-\mathrm{b}} \tau^{\mathrm{b}}}{1-\mathrm{b}}+0(1)\right)+0\left(\frac{1}{\mathrm{n}^{2-2 \mathrm{~b}}}\right) \\
& =\frac{\mathrm{a}}{\mathrm{h}_{\mathrm{n}}}\left(\frac{(1-\mathrm{b})^{2}}{(\mathrm{n} / \tau)^{2-2 b}}+0\left(\frac{1}{\mathrm{n}^{3-3 b}}\right)\right) \\
& \left(\frac{(\mathrm{n} / \tau)^{1-\mathrm{b}} \tau^{\mathrm{b}}}{1-\mathrm{b}}+0(1)\right) \int_{-1}^{1} \mathrm{k}^{2}(\mathrm{z}) \mathrm{dz}+0\left(\frac{1}{\mathrm{n}^{2-2 \mathrm{~b}}}\right) \\
& =a\left(\begin{array}{c}
\frac{(1-b) \tau^{b}}{(n / \tau)^{1-b} h_{n}}+0\left(\frac{1}{n^{2-2 b} h_{n}}\right) \\
+0\left(\frac{1}{n^{2-2 b} h_{n}}\right)+0\left(\frac{1}{n^{3-3 b} h_{n}}\right)
\end{array}\right) \\
& \int_{-1}^{1} k^{2}(z) d z+0\left(\frac{1}{n^{2-2 b}}\right) \\
& =\frac{\mathrm{a}(1-\mathrm{b}) \tau^{\mathrm{b}}}{(\mathrm{n} / \tau)^{1-\mathrm{b}} \mathrm{h}_{\mathrm{n}}} \int_{-1}^{1} \mathrm{k}^{2}(\mathrm{z}) \mathrm{dz}+0\left(\frac{1}{\mathrm{n}^{2-2 \mathrm{~b}} \mathrm{~h}_{\mathrm{n}}}\right) \\
& +0\left(\frac{1}{\mathrm{n}^{2-2 b} h_{n}}\right)+0\left(\frac{1}{n^{3-3 b} h_{n}}\right)+0\left(\frac{1}{n^{2-2 b}}\right) \\
& =\frac{a(1-b) \tau^{b}}{(n / \tau)^{1-b} h_{n}} \int_{-1}^{1} k^{2}(z) d z+0\left(\frac{1}{n^{2-2 b} h_{n}}\right)
\end{aligned}
$$

For $n \rightarrow \infty$.

With combining (40) and (47) into (30), then we obtained Eq. 48:

$$
\begin{aligned}
& \operatorname{Var} \bar{\lambda}_{\mathrm{c}, \mathrm{n}, \mathrm{k}}(\mathrm{s}) \\
& =0\left(\frac{1}{\mathrm{n}^{2-2 \mathrm{~b}} \mathrm{~h}_{\mathrm{n}}}\right)+\frac{\mathrm{a}(1-\mathrm{b}) \tau^{\mathrm{b}}}{(\mathrm{n} / \tau)^{1-\mathrm{b}} \mathrm{h}_{\mathrm{n}}} \int_{-1}^{1} \mathrm{k}^{2}(\mathrm{z}) \mathrm{dz} \\
& +0\left(\frac{1}{\mathrm{n}^{2-2 \mathrm{~b}} \mathrm{~h}_{\mathrm{n}}}\right)=\frac{\mathrm{a}(1-\mathrm{b}) \tau^{\mathrm{b}}}{(\mathrm{n} / \tau)^{1-\mathrm{b}} \mathrm{h}_{\mathrm{n}}} \int_{-1}^{1} \mathrm{k}^{2}(\mathrm{z}) \mathrm{dz}+0\left(\frac{1}{\mathrm{n}^{2-2 \mathrm{~b}} \mathrm{~h}_{\mathrm{n}}}\right)
\end{aligned}
$$

For $n \rightarrow \infty$ We considered that $\frac{1}{n^{1-b}}=0(1)$ for $n \rightarrow \infty$ then the right side of (48) becomes Eq. 49:

$$
\begin{aligned}
& =\frac{a(1-b) \tau^{b}}{(n / \tau)^{1-b} h_{n}} \int_{-1}^{1} k^{2}(z) d z+0\left(\frac{1}{n^{1-b} h_{n}}\right) \frac{1}{n^{1-b}} \\
& =\frac{a(1-b) \tau^{b}}{(n / \tau)^{1-b} h_{n}} \int_{-1}^{1} k^{2}(z) d z+o\left(\frac{1}{n^{1-b} h_{n}}\right)
\end{aligned}
$$

For $\mathrm{n} \rightarrow \infty$ Theorem 2 is proven.

Convergence of the estimator 1: From Eq. 10, it can be verified that $\mathrm{E}\left(\bar{\lambda}_{\mathrm{c}, \mathrm{n}, \mathrm{k}}(\mathrm{s})\right) \rightarrow \lambda_{\mathrm{c}}(\mathrm{s})$ for $\mathrm{n} \rightarrow \infty$ in other words $\bar{\lambda}_{\mathrm{c}, \mathrm{n}, \mathrm{k}}$ (s) is an unbiased estimator for $\lambda_{\mathrm{c}}(\mathrm{s})$. From Eq. 25 it can be verified that $\operatorname{Var} \bar{\lambda}_{c, n, k}(s) \rightarrow 0$ for $n \rightarrow \infty$ so that form these results we obtained MSE $\bar{\lambda}_{c, n, k}(s)$ for $\mathrm{n} \rightarrow \infty$.

\section{DISCUSSION}

The construction of the estimation for periodic component of the intensity function of a periodic Poisson process in the presence of power function trend can give Mean Square Error of this estimator asymptotically convergent to zero for $n \rightarrow \infty$.

\section{CONCLUSION}

We have constructed an estimator for periodic component of the intensity function of a periodic Poisson process in the presence of power function trend. In the estimation process, we do not use any assumption, except $\mathrm{N}$ is a Poisson process observed in bounded interval $[0, \mathrm{n}]$. From the estimation we get, Mean Square Error for the estimator convergent to zero for $\mathrm{n} \rightarrow \infty$.

There are two things of the development for this study. First, unlike the previous studies which the intensity function estimation only for periodic component with linear trend $(b=1)$, this study includes a power function trend $(0<b<1)$, because of many phenomena when the rate of the event increases by a power function of time. 
Second, in this study also extended to the more general case, we no longer use a uniform kernel function (when the probability density function is for uniform distribution random variables) but in this study (K.1) is a probability density function for arbitrary distribution.

\section{REFERENCES}

Helmers, R. and I.W. Mangku, 2000. Statistical estimation of poisson intensity function. Proceedings of the SEAM-GMU Intenational Conference on Mathematics and its Applications, Jul. 26-29, Yogyakarta, pp: 9-21.

Helmers, R. and I.W. Mangku, 2009. Estimating the intensity of a cyclic Poisson process in the presence of linear trend. Ann. Inst. Stat. Math., 61: 599-628. DOI: 10.1007/s10463-007-0160-2

Helmers, R. and R. Zitikis, 1999. On estimation of poisson intensity functions. Ann. Inst. Stat. Math., 51: 265-280. DOI: 10.1023/A:1003806107972

Helmers, R., 1995. on estimating the intensity of oilpollution in the North-Sea. CWI.

Helmers, R., I.W. Mangku and R. Zitikis, 2003. Consistent estimation of the intensity function of a cyclic poisson process. J. Multivariate Anal., 84: 19-39. DOI: $10.1016 / \mathrm{S} 0047-259 \mathrm{X}$ (02)00008-8
Helmers, R., I.W. Mangku and R. Zitikis, 2007. A nonparametric estimator for the doubly periodic poisson intensity function. Stat. Meth., 4: 481-492. DOI: 10.1016/j.stamet.2007.02.002

Mangku, I.W., 1999. Nearest Neighbor Estimation of the Intensity Function of a Cyclic Poisson Process. 1st Edn., CWI, Amsterdam, pp: 11.

Rachmawati, RN., 2008. Statistical properties of the estimator of periodic poisson process with power function trend. Undergraduate Thesis, Bogor Agricultural University.

Rahayu, M., 2008. Consistency of the estimator of the intensity function of a periodic poisson processes with power function trend. Undergraduate Thesis, Bogor Agricultural University.

Wheeden, R.L. and A. Zygmund, 1997. Measure and Integral: An Introduction to Real Analysis. 1st Edn., CRC Press, ISBN-10: 0824764994, pp: 288.

Yuliawati, L., 2008. Estimation of Global Intensity Function Periodic Poisson Processes with Power Function Trend. Undergraduate Thesis, Bogor Agricultural University. 